\title{
Geriatric assessment and intervention in older vulnerable patients undergoing surgery for colorectal cancer: a protocol for a randomised controlled trial (GEPOC trial)
}

Troels G. Dolin ${ }^{1,2^{*}}$ D, Marta Mikkelsen ${ }^{3}$, Henrik L. Jakobsen ${ }^{4}$, Tyge Nordentoft ${ }^{4}$, Trine S. Pedersen ${ }^{1}$, Anders Vinther ${ }^{5,6}$, Bo Zerahn ${ }^{7}$, Kirsten K. Vistisen ${ }^{3}$, Charlotte Suetta ${ }^{1,2,8,9}$, Dorte Nielsen ${ }^{3,9}$, Julia S. Johansen ${ }^{1,3,9}$ and Cecilia M. Lund ${ }^{1,2,9}$

\begin{abstract}
Background: The incidence of colorectal cancer (CRC) increases with age. Older patients are a heterogeneous group ranging from fit to frail with various comorbidities. Frail older patients with CRC are at increased risk of negative outcomes and functional decline after cancer surgery compared to younger and fit older patients. Maintenance of independence after treatment is rarely investigated in clinical trials despite older patients value it as high as survival. Comprehensive geriatric assessment (CGA) is an evaluation of an older persons' medical, psychosocial, and functional capabilities to develop an overall plan for treatment and follow-up. The beneficial effect of CGA is well documented in the fields of medicine and orthopaedic surgery, but evidence is lacking in cancer surgery. We aim to investigate the effect of CGA on physical performance in older frail patients undergoing surgery for CRC.

Methods: GEPOC is a single centre randomised controlled trial including older patients ( $\geq 65$ years) undergoing surgical resection for primary CRC. Frail patients ( $\leq 14 / 17$ points using the G8 screening tool) will be randomised 1:1 to geriatric intervention and exercise $(n=50)$ or standard of care along $(n=50)$ with their standard surgical procedure. Intervention includes preoperative CGA, perioperative geriatric in-ward review and postoperative followup. All patients in the intervention group will participate in a pre- and postoperative resistance exercise programme (twice/week, $2+12$ weeks). Primary endpoint is change in 30-s chair stand test. Assessment of primary endpoint will be performed by physiotherapists blinded to patient allocation. Secondary endpoints: changes in health related quality of life, physical strength and capacity (handgrip strength, gait speed and 6 min walking test), patient perceived quality of recovery, complications to surgery, body composition (Dual-energy X-ray absorptiometry and bioelectric impedance), serum biomarkers, readmission, length of stay and survival.

\footnotetext{
* Correspondence: Troels.gammeltoft.dolin@regionh.dk

'Department of Medicine, Copenhagen University Hospital, Herlev and Gentofte, Borgmester Ib Juuls Vej 1, DK-2730 Herlev, Denmark

${ }^{2}$ CopenAge - Copenhagen Center for Clinical Age Research, University of Copenhagen, Copenhagen, Denmark

Full list of author information is available at the end of the article
}

(c) The Author(s). 2021 Open Access This article is licensed under a Creative Commons Attribution 4.0 International License, which permits use, sharing, adaptation, distribution and reproduction in any medium or format, as long as you give appropriate credit to the original author(s) and the source, provide a link to the Creative Commons licence, and indicate if changes were made. The images or other third party material in this article are included in the article's Creative Commons licence, unless indicated otherwise in a credit line to the material. If material is not included in the article's Creative Commons licence and your intended use is not permitted by statutory regulation or exceeds the permitted use, you will need to obtain permission directly from the copyright holder. To view a copy of this licence, visit http://creativecommons.org/licenses/by/4.0/ The Creative Commons Public Domain Dedication waiver (http://creativecommons.org/publicdomain/zero/1.0/) applies to the data made available in this article, unless otherwise stated in a credit line to the data. 
(Continued from previous page)

Discussion: This ongoing trial will provide valuable knowledge on whether preoperative CGA and postoperative geriatric follow-up and intervention including an exercise program can counteract physical decline and improve quality of life in frail CRC patients undergoing surgery.

Trial registration: Prospectively registered at Clinicaltrials.gov NCT03719573 (October 2018).

Keywords: Colorectal cancer, Comprehensive geriatric assessment, Surgery, Frailty, Sarcopenia, Exercise

\section{Background}

Colorectal cancer (CRC) is the third most common cancer worldwide accounting for 1.8 million new cases in 2018 [1]. The incidence of CRC increases with age [2], with a median age at time of diagnosis of 70 years in Denmark [3]. As the population is getting older, an increasing number of older patients with CRC is expected in the future $[4,5]$.

The mainstay in treatment of localised CRC is resection [6]. Implementation of better perioperative care, minimal invasive surgery and enhanced recovery pathways have made cancer surgery available for a larger proportion of patients with CRC [7]. Surgery itself and complications from surgery increase the risk of functional decline and loss of independence which can be difficult to regain [8]. The burden of treatment as well as possible treatment outcomes are strong determinants of older patients' preferences [9]. Maintenance of independence and health related quality of life represent preferred treatment outcomes over standard oncological outcomes (e.g. disease free survival) for older patients [9-11]. In general, surgery is considered safe for older patients and the primary risk factor for poor surgical outcome, usually defined as surgical complications or survival, is not age but comorbidity [12], frailty [13, 14] and sarcopenia [15]. However, loss of independence is reported in 1 in 5 patients older than 65 years undergoing surgery for CRC $[8,16]$.

Frailty is recognised as an independent risk factor and has been described as "A medical syndrome with multiple causes and contributors that is characterized by diminished strength, endurance, and reduced physiologic function that increases an individual's vulnerability for developing increased dependency and/or death" [17]. Frail patients are more susceptible to adverse effects from external stressors such as surgery [13]. Identifying frail people is therefore crucial for more careful planning of surgery and treatment. Merely screening for frailty brings awareness of patient comorbidity and capacity and is associated with reduced mortality after surgery [18]. Frailty is not a permanent state but potentially reversible [19] since some comorbidities are modifiable, as are both physical capacity and nutritional status.

Comprehensive Geriatric assessment (CGA) is hitherto the most appropriate way to examine the overall health situation of the older frail patient [20]. CGA is a multidomain and usually multidisciplinary work tool used for systematic assessment of an older person's medical, psychosocial, as well as functional capabilities and limitations in order to develop an overall plan for interventions and follow-up [21]. CGA assesses known pathology and can uncover unrecognized health issues [22]. Furthermore, a prospective study has shown that CGA can assess the risk of postoperative complications in older patients undergoing elective CRC surgery [23]. Nonetheless, CGA is time consuming and may not be needed for all older patients. Several more rapid frailty screening tools have therefore been developed to identify which patients will benefit from a CGA [24]. The recommendations from the International Society for Geriatric Oncology (SIOG) from June 2014 [25] find the G8 questionnaire to be equally sensitive or superior when compared to other screening tools. For patients with an abnormal G8 score $(\leq 14 / 17)$ a full CGA is recommended [26, 27].

One of the most important factors leading to frailty is low muscle strength, functional performance and low muscle mass (i.e. sarcopenia) [17]. Progressive resistance training is the single most effective intervention to increase muscle strength, muscle mass and functional performance in healthy older individuals [28] and in different patient populations [29-32]. Physical exercise is therefore recommended by WHO [33] and is shown to be safe and beneficial among older patients with cancer $[34,35]$.

Yet, there is a need for randomised controlled trials investigating the effect of the CGA in cancer surgery with patient-centred outcomes as preferred by the older patients. Thus, the aim of the present study is to evaluate the effect of pre-operative CGA and physical exercise, post-operative geriatric in-ward review and exercise, and post-operative geriatric rehabilitation on patients' physical and functional performance.

\section{Methods/design \\ Trial design}

This is a randomised controlled trial (RCT) comparing a multidisciplinary intervention to standard of care in older frail patients with CRC undergoing curative intended surgery. The trial design follows the SPIRIT 
guidelines for randomised controlled trials. Trial design can be seen in Fig. 1 .

\section{Participants}

A total of 100 patients will be recruited from a single tertiary surgical centre at Herlev and Gentofte Hospital, Copenhagen University Hospital.

\section{Inclusion criteria}

For inclusion in the study, all the following must be fulfilled: (i) age $\geq 65$ years; (ii) frailty evaluated with a G8 questionnaire score $\leq 14 / 17$; (iii) scheduled for elective primary CRC surgical resection; and (iv) histologically proven adenocarcinoma.

\section{Exclusion criteria}

None of the following should apply if the patient is eligible for the study: (i) neoadjuvant treatment; (ii) severe physical disability that hinders training; and (iii) inability to give informed consent.

\section{Randomisation}

Randomisation is performed electronically in REDcap randomisation module from permuted blocks and stratified for Eastern Cooperative Oncology Group (ECOG) performance status. Patients will be randomized 1:1 to an intervention group and a control group.

\section{Management in both groups}

All patients will be evaluated at the multidisciplinary team (MDT) conference before surgery. Surgical

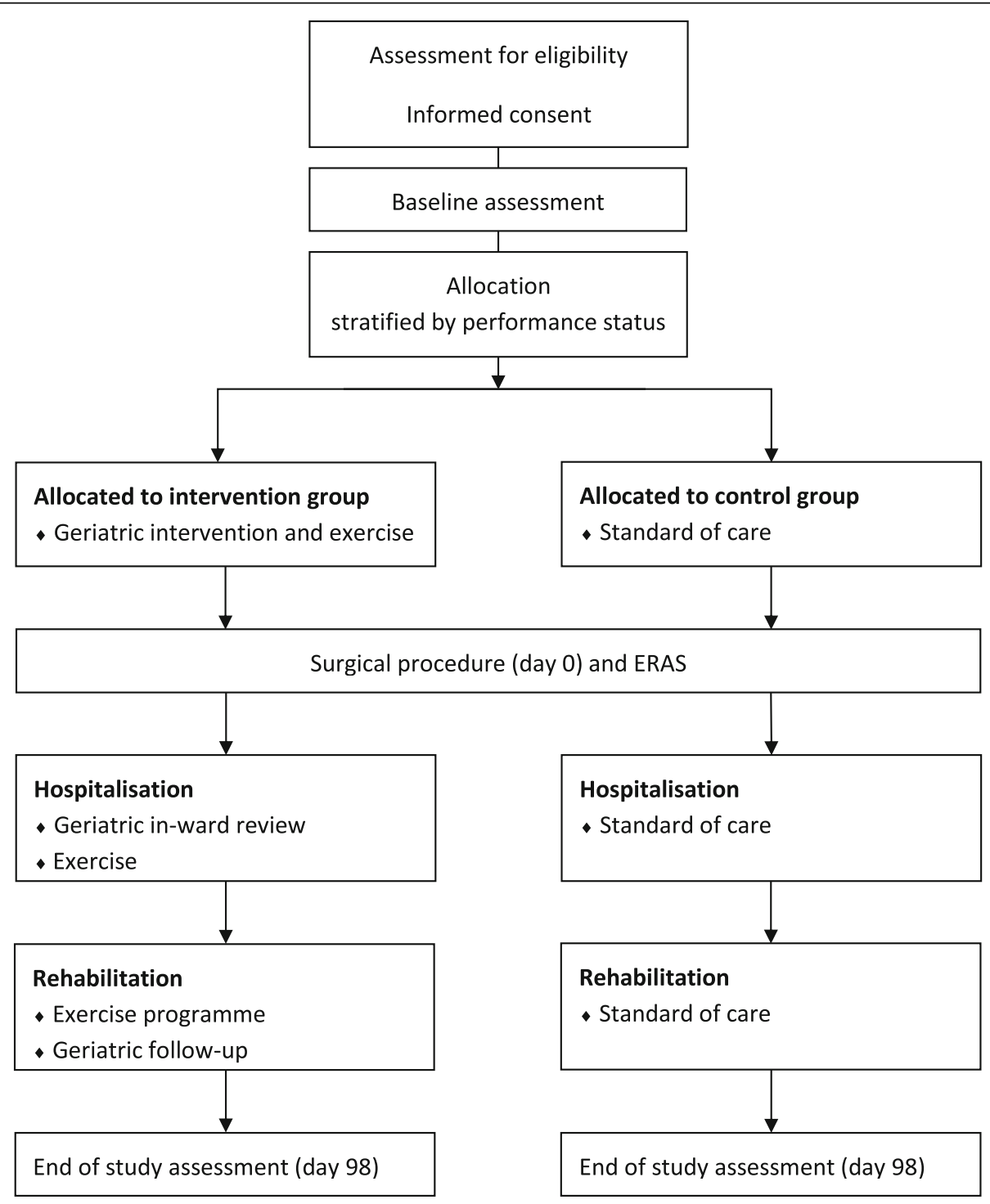

Fig. 1 GEPOC trial design. Abbreviation: ERAS Enhanced Recovery After Surgery 
resection is according to national guidelines [6] and follow the local Enhanced Recovery After Surgery (ERAS) programme with minimal invasive surgery [36]. The ERAS programme also includes pre-operative participation in the ERAS school where patients receive (i) education from a nurse specialist in preparation for surgery, (ii) education about nutrition from a dietician and (iii) education on mobilisation and lung physiotherapy from a physiotherapist. After surgery the ERAS programme includes early mobilisation and oral nutrition at the surgical ward. The geriatric intervention will not affect the surgical planning (e.g. laparoscopic or open surgery) nor the timing for surgery.

\section{Geriatric intervention}

All patients in the intervention group will receive a three-phased intervention: pre-operative-, perioperative- and post-operative. All phases consist of geriatric interventions including a physical exercise programme.

\section{Geriatric assessment and tailored interventions}

The pre-operative phase consists of a home visit with a CGA with tailored interventions on identified health issues. The contents of the geriatric assessment is based on recommendations from SIOG [37]. The following domains are assessed: comorbidity, medication, social status, cognition, psychological status, functional status and nutrition. Assessment tools and possible interventions can be seen in Supplement 1.

The peri-operative phase includes post-operative geriatric review in addition to surgical review in the surgical ward. This includes early detection and treatment of medical complications (e.g. delirium, infections, atrial fibrillation) and multidisciplinary issues (e.g. discharge planning, pain management, nutrition).

The post-operative phase: Geriatric follow-up on identified health problems if needed.

\section{Exercise programme}

All phases of the programme are supervised by experienced physiotherapists.

Pre- and post-operative phases: supervised groupbased exercise programme scheduled twice a week from inclusion until surgery. After a two-week post-operative pause the programme continues for further 12 consecutive weeks. The exercise programme is a 1 -h programme including $15 \mathrm{~min}$ warming up, $35 \mathrm{~min}$ of progressive resistance training followed by $10 \mathrm{~min}$ of cool down. The progressive resistance training focuses on large muscle groups and follows recommendations from American College of Sports Medicine [38-40]. The programme has previously been described by Mikkelsen et al. [41] The pre- and post-operative phases of exercise are both conducted in the Department of Physiotherapy and Occupational Therapy, Herlev Hospital.

Perioperative phase: patients will receive supervised training from physiotherapists throughout the stay when admitting on the surgical ward. The training programme is adapted to each individual in accordance with the surgical procedure (e.g. laparoscopic or laparotomic approach) and conferred with the surgical staff before each session. The exercise programme is conducted in the surgical ward. Details can be seen in Supplement 2 . After discharge patients will be provided with an activity tracker. They will be guided in setting goals in relation to activity throughout the study.

\section{Endpoints and measurements}

Baseline test prior to randomisation includes test of physical strength and capacity, questionnaire of quality of life and estimation of body composition. Study data are collected on the 2 . and 14. postoperative day, and at the end of study 14 weeks after surgery (Fig. 2).

\section{Primary endpoint}

The primary endpoint of the GEPOC study is change in 30-s Chair Stand Test (30s-CST) from baseline to 14 weeks after surgery. The 30s-CST measures a combination of muscle strength and endurance and is highly sensitive in older patients [42]. A high test-score is regarded as a predictor for functional independence in older patients [43-45] as it measures the ability to rise from a chair. The 30s-CST has also been used and tested in cancer patients $[46,47]$.

\section{Secondary endpoints}

\section{Muscle strength and functional capacity:} changes in hand grip strength, $6 \mathrm{~m}$ and $10 \mathrm{~m}$ habitual gait speed, 5-times-sit-to-stand and 6-minwalk-test. The 5-times-sit-to-stand is performed separately in accordance with latest guidelines for sarcopenia [48]. Changes in the physical tests will be reported from baseline at two and 14 weeks after surgery and changes from 2 weeks after surgery at 14 weeks after surgery. Additional information regarding the tests are described in Supplement 3. Cumulated Ambulation Score (CAS) [49] will be measured in the postoperative period, as a measurement of day-to-day basic mobility. For acute abdominal surgery a poor CAS is associated with pulmonary complications and low activity in the first postoperative week [50] and CAS has been shown to be predictive for discharge to a higher level of dependence [49]. CAS will be compared between groups and investigated as a predictor for poor postoperative outcome independent of patient 


\begin{tabular}{|c|c|c|c|c|c|c|}
\hline & Baseline & $\begin{array}{c}\text { Preoperative } \\
\text { period }\end{array}$ & Day of surgery & Hospitalisation & $\begin{array}{c}\text { Post-operative } \\
\text { visit }\end{array}$ & Study end \\
\hline Days from surgery & -14 & -14 to -1 & 0 & +1 to $x$ & +14 & +98 \\
\hline \multicolumn{7}{|l|}{ ENROLMENT: } \\
\hline Eligibility screen & $\mathrm{x}$ & & & & & \\
\hline Informed consent & $\mathrm{X}$ & & & & & \\
\hline Baseline assessment & $x$ & & & & & \\
\hline Allocation & $\mathrm{X}$ & & & & & \\
\hline \multicolumn{7}{|l|}{ INTERVENTIONS: } \\
\hline Home visit CGA & & $\mathrm{X}$ & & & & \\
\hline \multicolumn{7}{|l|}{ Geriatric ward } \\
\hline \multicolumn{7}{|l|}{ Exercise programme } \\
\hline \multicolumn{7}{|l|}{ Inpatient exercise } \\
\hline ERAS school & & $x$ & & & & \\
\hline \multicolumn{7}{|l|}{ CONTROLS: } \\
\hline ERAS school & & $x$ & & & & \\
\hline \multicolumn{7}{|l|}{ ASSESSMENTS: } \\
\hline Physical tests* & $x$ & & & & $x$ & $x$ \\
\hline CAS & & & & $\mathrm{X}$ & & \\
\hline Health related quality of life ${ }^{* *}$ & $\mathrm{x}$ & & & & $\mathrm{X}$ & $\mathrm{x}$ \\
\hline Quality of recovery-15 & & $x$ & & $x$ & $x$ & \\
\hline$B I$ & $\mathrm{x}$ & & & $\mathrm{X}$ & $x$ & $\mathrm{x}$ \\
\hline DXA & $X$ & & & & & $\mathrm{X}$ \\
\hline Serum biomarkers ${ }^{* * *}$ & $x$ & & & $x$ & & $x$ \\
\hline
\end{tabular}

Fig. 2 SPIRIT flow diagram. Abbreviations: CGA Comprehensive Geriatric Assessment, ERAS Enhanced Recovery After Surgery, CAS Cumulated Ambulation Score, BI Bioelectric Impedance, DXA Whole-body dual-energy X-ray absorptiometry. ${ }^{*}$ Physical tests: $30-\mathrm{s}$ chair stand test, $6+10-\mathrm{m}$ usual gait speed, handgrip strength, 5-times-sit-to-stand, six-minute-walk-test. ${ }^{* *}$ questionnaires: European Organization for Research and Treatment of Cancer Quality of Life Questionnaire Core 30 (EORTC QLQ-C30) + Elderly Cancer Patients Module (EORTC QLQ-ELD14). *** Serum biomarkers: Interleukin-6, YKL-40, C-reactive Protein, Olink panel, Growth Differentiation Factor $11+14$, Vitamin D

allocation to control or intervention group. All physical tests will be performed in the same sequence by a physiotherapist blinded to patients' allocation to control or intervention group.
2. Patient reported outcome measures: Patient's perspective of recovery in the perioperative period will be assessed with Quality of Recovery-15 (QoR15). QoR-15 is a patient reported outcome 
questionnaire that quantifies recovery from surgery and anaesthesia [51, 52].

Health related Quality of Life (HRQoL) will be assessed with European Organization for Research and Treatment of Cancer Quality of Life Questionnaire Core 30 (EORTC QLQ-C30) conjoined with the module for older patients with cancer (QLQ-ELD14). The questionnaires incorporate functional scales, symptom scales and a global health and quality-of-life scale [53, 54].

3. Postoperative complications $(\mathbf{3 0}+\mathbf{9 0}$ days): The occurrence of postoperative complication within $30+90$ days after surgical resection of CRC will be graded from 0 to 5 based on the classification system validated by Clavien and Dindo [55]. Further details are described in Supplement 4.

4. Body composition and sarcopenia: Whole-body dual-energy X-ray absorptiometry (DXA) scan (GE lunar iDXA, GE Healthcare Technologies, Madison, Wisconsin, U.S.) and bioelectrical impedance (BI) assessment (Body Composition Monitor, Fresenius Medical Care, Bad Homburg v.d.H., Germany) will be used to measure body composition. Sarcopenia will be reported according to the revised guidelines from the European Working Group on Sarcopenia in Older People [48].

5. Length of stay and readmission rate: The postoperative length of stay defined from the day of surgical resection of CRC to the day of discharge from the surgical ward will be calculated.

Readmission rate is defined as any unplanned hospitalisation within 30 and 90 days of discharge is registered.

6. Initiation and completion of adjuvant chemotherapy: Patients will be treated with adjuvant chemotherapy according to national guidelines if indication for chemotherapy is found [56]. Type of chemotherapy, dose, number of series and adverse events are registered.

7. Survival and mortality: Cancer- and treatmentrelated mortality or other cause of mortality will be registered throughout the study and a during a follow-up period of 5 years.

8. Serum biomarkers: Blood samples will be taken 13 days before surgery, at the first and second postoperative day and at the end of study. Serum biomarkers will be analysed as part of cooperative research project REBECCA ("Biomarkers for patients with colorectal cancer - providing new information on diagnosis, treatment effect, sideeffects and prognosis" (translated from Danish)). The REBECCA study is approved by the Regional Ethics Committee (H-2-2013-078) and the Danish
Data Protection Agency (jr. nr. 2007-58-0015; HEH-2014-044; I-suite nr. 02771 and PACTIUS P2019-614).

To investigate the influence of CGA and CGA-based exercise for older frail patients with CRC the following serum biomarkers will be measured: $\mathrm{C}$-reactive protein (CRP), interleukin-6 (IL-6), YKL-40, Growth Differentiation Factor (GDF) 11 and 15, vitamin D and the Olink immune-oncology panel [57] (a panel of 92 serum proteins proteins associated with inflammation and immunology and cancer). Further details are given in Supplement 5.

\section{Statistical analysis \\ Sample size}

Calculation of statistical power is based on the estimated effect of CGA based interventions on physical performance. According to a previous study focusing on patients with osteoarthritis, the clinically relevant change in the $30 \mathrm{~s}-\mathrm{CST}$ was set at 2.6 repetitions [58]. Based on results from prior studies focusing on patients with advanced cancer, a standard deviation (SD) of around 3 has been reported in the 30s-CST $[59,60]$. To be able to detect a difference of 2.6 repetitions in the between-group difference in the 30s-CST at the 12-week assessment and to obtain a type I error rate of $5 \%$ and a power of $90 \%$, a sample size of 29 patients per study arm will be needed. To account for an expected dropout rate of $\sim 40 \%$, we decided to increase this number to a group size of 50 . Thus, the aim will be to include a total of 100 patients in the study.

\section{Quantitative data}

Results from physical tests, body composition measures, questionnaires and serum biomarkers will be reported as means and standard deviations (SD) or as median and IQR, as appropriate. Change over time in ordinal categorical values will be evaluated by a trend test using logistic regression. In-group and between- group differences in continuous-level data, will be performed using independent $T$-tests or the nonparametric MannWhitney $\mathrm{U}$ and Wilcoxon tests, depending on their distribution. Survival analyses will be conducted using Kaplan-Meier method, competing risk analyses and Cox regression analyses. Using the Kaplan-Meier method the cancer-specific mortality and overall survival (OS) will be assessed for the intervention group and the control group, and comparison in survival between groups will be assessed using the log-rank test.

Statistical analyzes will be performed by a statistician in collaboration with the primary investigator (TGD) using R Version 1.0.153. 


\section{Trial status}

The trial started in February 2019. Per primary January 2021124 patients have been screened for frailty, 59 have been frail according to G8. 36 patients have been included. Amendments to the protocol have been made due to a lower rate of frail patients and a lower recruitment rate than anticipated. Age for inclusion have been lowered from 70 to 65 years (July 2019) and eligibility of patients with rectum cancer have been included (November 2019).

\section{Discussion}

The present study is a RCT evaluating the effect of geriatric intervention in frail older patients undergoing surgery for primary CRC. The development of ERAS pathways and minimal invasive surgical technique have made surgery feasible for a broader population, including older patients. However, treatment decisions made today are often based on studies with younger and fitter patients, as the older frail population is often underrepresented in clinical trials [61]. Apart from observational studies [7], there is an urgent need for interventional studies focusing on patient-relevant outcomes as treatment goals for the older frail patients differ from their younger counterparts with an increased emphasis on independence, quality of life and physical capacity [9].

The CGA is a well-known tool in the intervention of older frail patients. In a meta-analysis of randomised trials, CGA-based interventions in patients admitted to the hospital for medical conditions increased the likelihood of being alive and living at home 6 months after hospital discharge [20]. The positive effect was greatest for geriatric wards, but geriatric teams also had a positive effect.

In orthopaedic surgery a beneficial effect in terms of 4 and 12 month physical function and increased independence was found for older patients with hip fractures randomised to comprehensive geriatric care compared to usual orthopaedic care [62]. For patients undergoing elective surgery, the incorporation of pre-operative CGA and post-operative inpatient geriatric review is seen in the UK with the Perioperative care of Older people undergoing Surgery (POPS) service. The POPS model was evaluated in a pre- and post-study and showed fewer postoperative medical complications, fewer multidisciplinary issues and a reduced length of hospitalisation [63]. The POPS concept was subsequently proved effective in a RCT of patients undergoing elective aorta or lower limb vascular surgery [64]. The RCT showed significantly reduced length of stay in the CGA group compared to standard care. The study did not use postoperative geriatric inpatient review but created a postoperative care plan providing advice on prevention and management of postoperative complications.
Few studies have investigated the effect of CGA in cancer surgery. In a recent Norwegian RCT, Ommundsen et al. [65] investigated the effect of preoperative geriatric intervention on postoperative complications (grade II-IV Clavien-Dindo classification) in patients undergoing elective surgery for CRC. No immediate difference was found between groups. When adjusted for prespecified prognostic factors, there was a statistically significant difference in favour of the intervention group when all complications (grade I-V) were taken into account. The study did not use post-operative geriatric inpatient review but made the geriatric assessment available to the surgical team and made general recommendations regarding medical and multidisciplinary issues (e.g. delirium and mobilisation). The study did not investigate the effect of CGA on functional status, quality of life or independence.

Older frail patients are prone to medical complications and loss of independence after surgery even if provided with optimal pre-operative care. Therefore, a major strength of the GEPOC study is expected to be the planned post-operative geriatric inpatient review. Furthermore, the broad collection of outcomes both in terms of physical testing, patient reported outcomes, body composition and serum biomarkers allow for a detailed insight in the effect of CGA based intervention in the population. To our knowledge no RCTs have been published investigating the effect of both pre-operative CGA and post-operative geriatric inpatient review for patients undergoing cancer surgery.

The CGA-based exercise programme is delivered throughout the GEPOC study. The pre-operative part is in line with the increasing interest in prehabilitation in the field of perioperative medicine. Prehabilitation is described by Carli et al. as "the process of enabling patients to withstand the stress of surgery through augmenting functional capacity" [66]. Prehabilitation focuses on preconditioning through exercise, nutrition, smoking cessation and anxiety reducing elements. A meta-analysis has shown effect on lean body mass [67], but results are still lacking in important outcomes e.g. complications to surgery, HRQOL, independence and physical capacity. Carli et al. showed no difference between groups in a RCT published in 2020 comparing prehabilitation to rehabilitation [68] but larger international studies are ongoing [69]. In the GEPOC study, the preconditioning period is short due to Danish cancer care plans which ensure surgery within 2 weeks from diagnosis. This challenges the ability to detect a measurable effect of the pre-operative exercise programme. However, the pre-operative physical exercise serves to improve neuromuscular adaptation and enable patients to get familiar with the exercise facilities and the training programme, thus facilitating a safe and effective return in the rehabilitation period. As 
older frail patients often have complex pathology going into surgery, a strength of CGA-based exercise in the GEPOC study is the assessment and individually tailored interventions ensuring a more personalised and holistic treatment approach also targeting possible modifiable barriers for exercise (e.g. dizziness as a result of too aggressive antihypertensive treatment). Furthermore, the effect of rehabilitation is well proven, and we hypothesize that combining prehabilitation and rehabilitation rather than only applying one of these would serve as a more beneficial treatment option for frail older patients. A limitation to this study setup is that it is unable to evaluate the effect of either prehabilitation or rehabilitation as a separate intervention.

Sarcopenia is reported in the GEPOC study and is regarded as one of the physical drivers of frailty. It is acknowledged as a separate entity characterised by muscle failure [70]. This is primarily defined by low muscle strength and can be confirmed by reduced muscle quantity and/or quality. If muscle functioning is impaired as well, sarcopenia is considered severe [48]. Sarcopenia has multiple contributing factors including the ageing process itself, insufficient nutrition, inactivity (sedentary lifestyle) and chronic diseases like cancer. The association between frailty and sarcopenia has not been fully elucidated, but they are linked with many of the same clinical outcomes e.g. low survival and complications to surgery [71]. It has also been suggested that the role of an aged immune system, with the age-related decline in immune function and a state of chronic inflammation, could play at potential role in the pathophysiology of both frailty and sarcopenia [72]. Interventions for treatment of sarcopenia are often multimodal with resistance exercise and diet advice on adequate protein and calorie intake $[73,74]$ which also are domains addressed in the CGA. In the GEPOC study we perform serial assessments of sarcopenia throughout the study period allowing for obtaining valuable information on the effect of CGA on sarcopenia. Identifying and treating sarcopenia could be a key factor in preventing negative implications of frailty in an ageing population of patients with cancer.

\section{Supplementary Information}

The online version contains supplementary material available at https://doi. org/10.1186/s12877-021-02045-9.

Additional file 1 Supplementary file 1. Contents of the comprehensive geriatric assessment before surgery. Supplementary file 2. Exercise programme during hospitalisation. Supplementary file 3. Endpoints - Physical function tests. Supplementary file 4. Clavien and Dindo classification system for postoperative complication. Supplementary file $\mathbf{5}$. Serum biomarkers.

\section{Abbreviations}

ADL: Activities of daily living; BMI: Body mass index; BI: Bioelectrical impedance; CAM : Confusion Assessment Method; CAS: Cumulated
Ambulation Score; CGA: Comprehensive Geriatric Assessment; CRP: C-reactive protein; CRC: Colorectal cancer; DXA: Dual-energy X-ray absorptiometry scan; EORTC: European Organisation for Research and Treatment of Cancer; EORTC QLQ-C30: European Organisation for Research and Treatment of Cancer Quality of Life Questionnaire- C30; GDF 11: Growth Differentiation Factor 11; GDF 15: Growth Differentiation Factor 15; Gl: Geriatric intervention; HST: Handgrip strength test; IL-6: Interleukin 6; IQR: Interquartile range; MMSE: Mini Mental State Examination; PA: Physical activity; PROM: Patientreported outcome measure; PRT: Progressive resistance training; PS: Performance status; RM: Repetition maximum; SD: Standard Deviation; SIOG: International Society of Geriatric Oncology; START: Screening Tool to Alert doctors to Right Treatment; STOPP: Screening Tool Older Person's Prescriptions; QOL: Quality of life; 30s-CST: 30-s chair stand test; 6MGS: 6-m gait speed; 6MWT: Six-minute-walk-test

\section{Acknowledgements}

Not applicable.

\section{Authors' contributions}

TGD, MKM, HLJ, TSP, AV, CS, DN, JSJ and CML made substantial contributions to the conception and design of the work. TGD, MKM, TN, AV, BZ, KKV, CS, DN, JSJ and CML have drafted the work or substantively revised it. All authors read and approved the final manuscript.

\section{Funding}

The Velux foundation is funding salary to corresponding author TGD and CL. Danielsen's Foundation and Beckett Foundation have given financial support to testing by blinded physiotherapist and the exercise intervention. The design, management, analysis and reporting of the study are entirely independent.

\section{Availability of data and materials}

Not Applicable.

\section{Ethics approval and consent to participate}

The study protocol was approved by the Scientific Ethics Review Committee of the Capital Region of Denmark in January 2019 (ref. H18051304).

Amendments have been accepted July 2019 (ref. 69163) and November 2019 (ref. 70778). The study is approved by Danish Data Protection Agency in December 2018 (ref. VD-2018-388). The trial has been registered on the clinicaltrials.org website under the identification number NCT03719573 on October 2018, prior to inclusion start. The study complies with the Declaration of Helsinki [75]. All participants will have to sign an informed consent form.

Consent for publication

Not applicable.

\section{Competing interests}

The authors declare that they have no competing interests.

\section{Author details}

${ }^{1}$ Department of Medicine, Copenhagen University Hospital, Herlev and Gentofte, Borgmester Ib Juuls Vej 1, DK-2730 Herlev, Denmark. ${ }^{2}$ CopenAge Copenhagen Center for Clinical Age Research, University of Copenhagen, Copenhagen, Denmark. ${ }^{3}$ Department of Oncology, Copenhagen University Hospital, Herlev and Gentofte, Copenhagen, Denmark. ${ }^{4}$ Department of Surgery, Copenhagen University Hospital, Herlev and Gentofte, Copenhagen, Denmark. ${ }^{5}$ Department of Physiotherapy and Occupational Therapy, Copenhagen University Hospital, Herlev and Gentofte, Copenhagen, Denmark. ${ }^{6}$ Hospital Secretariat and Communications, Research, Copenhagen University Hospital, Herlev and Gentofte, Copenhagen, Denmark.

${ }^{7}$ Department of Clinical Physiology and Nuclear Medicine, Copenhagen University Hospital, Herlev and Gentofte, Copenhagen, Denmark. ${ }^{8}$ Department of Geriatric and Palliative Medicine, Copenhagen University Hospital, Bispebjerg and Frederiksberg Hospital, Copenhagen, Denmark. ${ }^{9}$ Department of Clinical Medicine Faculty of Health and Medical Sciences, University of Copenhagen, Copenhagen, Denmark. 
Received: 3 September 2020 Accepted: 21 January 2021

\section{Published online: 30 January 2021}

\section{References}

1. Siegel RL, Miller KD, Jemal A. Cancer statistics, 2020. CA Cancer J Clin. 2020; 70(1):7-30.

2. Keum N, Giovannucci E. Global burden of colorectal cancer: emerging trends, risk factors and prevention strategies. Nat Rev Gastroenterol Hepatol. 2019;16(12):713-32.

3. Danckert B, Ferlay J, Engholm G, Hansen HL, Johannesen TB, Khan S, Køtlum JE, Ólafsdóttir E, Schmidt LKH, Virtanen A and Storm HH. NORDCAN: Cancer Incidence, Mortality, Prevalence and Survival in the Nordic Countries, Version 8.2 (26.03.2019). Association of the Nordic Cancer Registries. Danish Cancer Society. Available from http://www.ancr.nu. Accessed 6 June 2020.

4. Parry C, Kent EE, Mariotto AB, Alfano CM, Rowland JH. Cancer survivors: a booming population. Cancer Epidemiol Biomarkers Prevention. 2011;20(10): 1996-2005.

5. Bluethmann SM, Mariotto AB, Rowland JH. Anticipating the "silver tsunami": prevalence trajectories and comorbidity burden among older Cancer survivors in the United States. Cancer Epidemiol Biomarkers Prevention. 2016;25(7):1029-36.

6. DCCG NkR. Kirurgisk behandling af cancer coli. 2019

7. Montroni I, Rostoft S, Spinelli A, Van Leeuwen BL, Ercolani G, Saur NM, et al. GOSAFE - geriatric oncology surgical assessment and functional rEcovery after surgery: early analysis on 977 patients. J Geriatr Oncol. 2020;11(2):24455.

8. Lawrence VA, Hazuda HP, Cornell JE, Pederson T, Bradshaw PT, Mulrow CD, et al. Functional independence after major abdominal surgery in the elderly. J Am Coll Surg. 2004;199(5):762-72.

9. Fried TR, Bradley EH, Towle VR, Allore H. Understanding the treatment preferences of seriously ill patients. N Engl J Med. 2002;346(14):1061-6.

10. Mohile SG, Hurria A, Cohen HJ, Rowland JH, Leach CR, Arora NK, et al. Improving the quality of survivorship for older adults with cancer. Cancer. 2016;122(16):2459-568.

11. Banks E, Byles JE, Gibson RE, Rodgers B, Latz IK, Robinson IA, et al. Is psychological distress in people living with cancer related to the fact of diagnosis, current treatment or level of disability? Findings from a large Australian study. Med J Aust. 2010;193(5 Suppl):S62-7.

12. Leung JM, Dzankic S. Relative importance of preoperative health status versus intraoperative factors in predicting postoperative adverse outcomes in geriatric surgical patients. J Am Geriatr Soc. 2001;49(8):1080-5.

13. Ommundsen N, Wyller TB, Nesbakken A, Jordhoy MS, Bakka A, Skovlund E, et al. Frailty is an independent predictor of survival in older patients with colorectal cancer. Oncologist. 2014;19(12):1268-75.

14. Makary MA, Segev DL, Pronovost PJ, Syin D, Bandeen-Roche K, Patel P, et al. Frailty as a predictor of surgical outcomes in older patients. J Am Coll Surg. 2010;210(6):901-8.

15. Simonsen $C$, de Heer $P$, Bjerre ED, Suetta $C$, Hojman $P$, Pedersen BK, et al. Sarcopenia and postoperative complication risk in gastrointestinal surgical oncology: a meta-analysis. Ann Surg. 2018;268(1):58-69.

16. Gearhart SL, Do EM, Owodunni O, Gabre-Kidan AA, Magnuson T. Loss of Independence in older patients after operation for colorectal Cancer. J Am Coll Surg. 2020;230(4):573-82

17. Morley JE, Vellas B, van Kan GA, Anker SD, Bauer JM, Bernabei R, et al. Frailty consensus: a call to action. J Am Med Dir Assoc. 2013;14(6):392-7.

18. Hall DE, Arya S, Schmid KK, Carlson MA, Lavedan P, Bailey TL, et al. Association of a Frailty Screening Initiative with Postoperative Survival at 30, 180, and 365 days. JAMA Surg. 2017;152(3):233-40.

19. Cameron ID, Fairhall N, Langron C, Lockwood K, Monaghan N, Aggar C, et al. A multifactorial interdisciplinary intervention reduces frailty in older people: randomized trial. BMC Med. 2013;11:65.

20. Ellis G, Whitehead MA, Robinson D, O'Neill D, Langhorne P. Comprehensive geriatric assessment for older adults admitted to hospital: meta-analysis of randomised controlled trials. BMJ. 2011;343:d6553.

21. Rubenstein LZ, Stuck AE, Siu AL, Wieland D. Impacts of geriatric evaluation and management programs on defined outcomes: overview of the evidence. J Am Geriatrics Society. 1991;39(9 Pt 2):8S-16S discussion 7S-8S.

22. Parker SG, McCue P, Phelps K, McCleod A, Arora S, Nockels K, et al. What is comprehensive geriatric assessment (CGA)? An umbrella review. Age Ageing. 2018;47(1):149-55.
23. Kristjansson SR, Nesbakken A, Jordhoy MS, Skovlund E, Audisio RA, Johannessen $\mathrm{HO}$, et al. Comprehensive geriatric assessment can predict complications in elderly patients after elective surgery for colorectal cancer: a prospective observational cohort study. Crit Rev Oncol Hematol. 2010; 76(3):208-17.

24. Kenig J, Zychiewicz B, Olszewska U, Richter P. Screening for frailty among older patients with cancer that qualify for abdominal surgery. J Geriatr Oncol. 2015;6(1):52-9.

25. Decoster L, Van Puyvelde K, Mohile S, Wedding U, Basso U, Colloca G, et al. Screening tools for multidimensional health problems warranting a geriatric assessment in older cancer patients: an update on SIOG recommendations. Ann Oncol. 2015;26(2):288-300.

26. Leo S, Accettura C, Gnoni A, Licchetta A, Giampaglia M, Mauro A, et al. Systemic treatment of gastrointestinal cancer in elderly patients. J Gastrointest Cancer. 2013:44(1):22-32.

27. Bellera CA, Rainfray M, Mathoulin-Pelissier S, Mertens C, Delva F, Fonck M, et al. Screening older cancer patients: first evaluation of the G-8 geriatric screening tool. Ann Oncol. 2012;23(8):2166-72.

28. Liu CJ, Latham NK. Progressive resistance strength training for improving physical function in older adults. Cochrane Database Systematic Reviews. 2009;2009(3):Cd002759.

29. Zimmer P, Trebing S, Timmers-Trebing U, Schenk A, Paust R, Bloch W, et al. Eight-week, multimodal exercise counteracts a progress of chemotherapyinduced peripheral neuropathy and improves balance and strength in metastasized colorectal cancer patients: a randomized controlled trial. Supportive Care Cancer. 2018:26(2):615-24.

30. Suetta C, Magnusson SP, Rosted A, Aagaard P, Jakobsen AK, Larsen LH, et al. Resistance training in the early postoperative phase reduces hospitalization and leads to muscle hypertrophy in elderly hip surgery patients--a controlled, randomized study. J Am Geriatr Soc. 2004;52(12):2016-22.

31. Parmenter BJ, Mavros Y, Ritti Dias R, King S, Fiatarone SM. Resistance training as a treatment for older persons with peripheral artery disease: a systematic review and meta-analysis. Br J Sports Med. 2020;54(8):452-61.

32. Hagstrom AD, Marshall PW, Lonsdale C, Papalia S, Cheema BS, Toben C, et al. The effect of resistance training on markers of immune function and inflammation in previously sedentary women recovering from breast cancer: a randomized controlled trial. Breast Cancer Res Treat. 2016;155(3): 471-82.

33. WHO. Global Recommendations on physical activity for health WHO Press. 2010.

34. Burhenn PS, Bryant AL, Mustian KM. Exercise promotion in geriatric oncology. Curr Oncol Rep. 2016;18(9):58.

35. Medicine ACOS. ACSM's guidelines for exercise testing and prescription. 2016.

36. Kehlet H. Fast-track colorectal surgery. Lancet. 2008;371(9615):791-3.

37. Wildiers H, Heeren P, Puts M, Topinkova E, Janssen-Heijnen ML, Extermann $M$, et al. International Society of Geriatric Oncology consensus on geriatric assessment in older patients with cancer. J Clin Oncol. 2014;32(24):2595603.

38. American College of Sports Medicine position stand. Progression models in resistance training for healthy adults. Med Sci Sports Exerc. 2009:41(3):687-708,

39. Schmitz KH, Courneya KS, Matthews C, Demark-Wahnefried W, Galvao DA, Pinto BM, et al. American College of Sports Medicine roundtable on exercise guidelines for cancer survivors. Med Sci Sports Exerc. 2010;42(7): 1409-26.

40. Chodzko-Zajko WJ, Proctor DN, Fiatarone Singh MA, Minson CT, Nigg CR, Salem GJ, et al. American College of Sports Medicine position stand. Exercise and physical activity for older adults. Med Sci Sports Exerc. 2009; 41(7):1510-30.

41. Mikkelsen MK, Lund CM, Vinther A, Tolver A, Ragle AM, Johansen JS, et al. Engaging the older cancer patient; patient activation through counseling, exercise and mobilization - pancreatic, biliary tract and lung cancer (PACEMobil-PBL) - study protocol of a randomized controlled trial. BMC Cancer. 2018;18(1):934.

42. Suetta C, Haddock B, Alcazar J, Noerst T, Hansen OM, Ludvig H, et al. The Copenhagen sarcopenia study: lean mass, strength, power, and physical function in a Danish cohort aged 20-93 years. J Cachexia Sarcopenia Muscle. 2019:10(6):1316-29.

43. Jones CJ, Rikli RE, Beam WC. A 30-s chair-stand test as a measure of lower body strength in community-residing older adults. Res Q Exerc Sport. 1999; 70(2):113-9 
44. Rikli REJC. Development and validation of a functional fitness test for community-residing older adults. J Aging Phys Act. 1999;7:129-61.

45. Rikli RE, Jones CJ. Development and validation of criterion-referenced clinically relevant fitness standards for maintaining physical independence in later years. Gerontologist. 2013;53(2):255-67.

46. Loenbro S DU, Primdahl, Overgaards J, Overgaard K. . Feasibility and efficacy of progressive resistance training and dietary supplements in radiotherapy treated head and neck cancer patients -- the DAHANCA 25A study. . Acta Oncol 2013;52(2):310-318.

47. Østergren P, Ragle AM, Jakobsen H, Klausen TW, Vinther A, Sønksen J. Group-based exercise in daily clinical practice to improve physical fitness in men with prostate cancer undergoing androgen deprivation therapy: study protocol. BMJ Open. 2016;6(6):e011460.

48. Cruz-Jentoft AJ, Bahat G, Bauer J, Boirie Y, Bruyere O, Cederholm T, et al. Sarcopenia: revised European consensus on definition and diagnosis. Age Ageing. 2019;48(1):16-31.

49. Foss NB, Kristensen MT, Kehlet $H$. Prediction of postoperative morbidity, mortality and rehabilitation in hip fracture patients: the cumulated ambulation score. Clin Rehabil. 2006;20(8):701-8.

50. Jonsson LR, Ingelsrud LH, Tengberg LT, Bandholm T, Foss NB, Kristensen MT. Physical performance following acute high-risk abdominal surgery: a prospective cohort study. Canadian J Surg. 2018;61(1):42-9.

51. Kleif J, Waage J, Christensen KB, Gogenur I. Systematic review of the QoR-15 score, a patient- reported outcome measure measuring quality of recovery after surgery and anaesthesia. Br J Anaesth. 2018;120(1):28-36.

52. Stark PA, Myles PS, Burke JA. Development and psychometric evaluation of a postoperative quality of recovery score: the QoR-15. Anesthesiology. 2013; 118(6):1332-40.

53. Aaronson NK, Ahmedzai S, Bergman B, Bullinger M, Cull A, Duez NJ, et al. The European Organization for Research and Treatment of Cancer QLQ-C30: a quality-of-life instrument for use in international clinical trials in oncology. J Natl Cancer Inst. 1993;85(5):365-76.

54. Papamichael D, Audisio RA, Glimelius B, de Gramont A, Glynne-Jones R, Haller D, et al. Treatment of colorectal cancer in older patients: International Society of Geriatric Oncology (SIOG) consensus recommendations 2013. Ann Oncol. 2015;26(3):463-76.

55. Dindo D, Demartines N, Clavien PA. Classification of surgical complications: a new proposal with evaluation in a cohort of 6336 patients and results of a survey. Ann Surg. 2004;240(2):205-13.

56. (DCCG) DCCG. 2018 [current guidelines for adjuvant treatment for colorectal cancer in Denmark (in Danish)]. Available from: https://dccg.dk/ retningslinjer/kolorektal-cancer/\#1502740758880-76f8c030-6fe2.

57. AB OP. Olink immuno-oncology panel https://www.olink.com/content/ uploads/2019/06/Olink-Immuno-Oncology-Validation-Data-v2.1.pdf [2.1: [Available from: https://www.olink.com/content/uploads/2019/06/OlinkImmuno-Oncology-Validation-Data-v2.1.pdf.

58. Wright AA, Cook CE, Baxter GD, Dockerty JD, Abbott JH. A comparison of 3 methodological approaches to defining major clinically important improvement of 4 performance measures in patients with hip osteoarthritis. J Orthop Sports Phys Ther. 2011;41(5):319-27.

59. Litterini AJ, Fieler VK, Cavanaugh JT, Lee JQ. Differential effects of cardiovascular and resistance exercise on functional mobility in individuals with advanced cancer: a randomized trial. Arch Phys Med Rehabil. 2013; 94(12):2329-35.

60. Oldervoll LM, Loge JH, Lydersen S, Paltiel H, Asp MB, Nygaard UV, et al. Physical exercise for cancer patients with advanced disease: a randomized controlled trial. Oncologist. 2011;16(11):1649-57.

61. Scher KS, Hurria A. Under-representation of older adults in cancer registration trials: known problem, little progress. J Clin Oncol. 2012;30(17):2036-8.

62. Prestmo A, Hagen G, Sletvold O, Helbostad JL, Thingstad P, Taraldsen K, et al. Comprehensive geriatric care for patients with hip fractures: a prospective, randomised, controlled trial. Lancet. 2015;385(9978):1623-33.

63. Harari D, Hopper A, Dhesi J, Babic-Illman G, Lockwood L, Martin F. Proactive care of older people undergoing surgery ('POPS'): designing, embedding, evaluating and funding a comprehensive geriatric assessment service for older elective surgical patients. Age Ageing. 2007;36(2):190-6.

64. Partridge JS, Harari D, Martin FC, Peacock JL, Bell R, Mohammed A, et al. Randomized clinical trial of comprehensive geriatric assessment and optimization in vascular surgery. Br J Surg. 2017;104(6):679-87.

65. Ommundsen N, Wyller TB, Nesbakken A, Bakka AO, Jordhoy MS, Skovlund E, et al. Preoperative geriatric assessment and tailored interventions in frail older patients with colorectal cancer: a randomized controlled trial. Colorectal Dis. 2018;20(1):16-25.

66. Carli F, Minnella EM. Preoperative functional assessment and optimization in surgical patient: changing the paradigm. Minerva Anestesiol. 2017;83(2):2148.

67. Gillis C, Fenton TR, Sajobi TT, Minnella EM, Awasthi R, Loiselle SE, et al. Trimodal prehabilitation for colorectal surgery attenuates post-surgical losses in lean body mass: A pooled analysis of randomized controlled trials. Clinical Nutrition (Edinburgh, Scotland). 2018;38(3):1053-60.

68. Carli F, Bousquet-Dion G, Awasthi R, Elsherbini N, Liberman S, Boutros M, et al. Effect of multimodal Prehabilitation vs postoperative rehabilitation on 30-day postoperative complications for frail patients undergoing resection of colorectal Cancer: a randomized clinical trial. JAMA Surg. 2020;155(3):23342.

69. van Rooijen S, Carli F, Dalton S, Thomas G, Bojesen R, Le Guen M, et al. Multimodal prehabilitation in colorectal cancer patients to improve functional capacity and reduce postoperative complications: the first international randomized controlled trial for multimodal prehabilitation. BMC Cancer. 2019;19(1):98.

70. Anker SD, Morley JE, von Haehling S. Welcome to the ICD-10 code for sarcopenia. J Cachexia Sarcopenia Muscle. 2016;7(5):512-4.

71. Wagner D, DeMarco MM, Amini N, Buttner S, Segev D, Gani F, et al. Role of frailty and sarcopenia in predicting outcomes among patients undergoing gastrointestinal surgery. World J Gastrointest Surg. 2016;8(1):27-40.

72. Wilson D, Jackson T, Sapey E, Lord JM. Frailty and sarcopenia: the potential role of an aged immune system. Ageing Res Rev. 2017;36:1-10.

73. Ali S, Garcia JM. Sarcopenia, cachexia and aging: diagnosis, mechanisms and therapeutic options - a mini-review. Gerontology. 2014;60(4):294-305.

74. Dunne RF et al. "Cachexia and Sarcopenia in Older Adults with Cancer: A Comprehensive Review." Cancers. 2019;11(12):1861. https://doi.org/10.3390/ cancers 11121861

75. World Medical Association Declaration of Helsinki. ethical principles for medical research involving human subjects. Jama. 2013;310(20):2191-4.

\section{Publisher's Note}

Springer Nature remains neutral with regard to jurisdictional claims in published maps and institutional affiliations.
Ready to submit your research? Choose BMC and benefit from:

- fast, convenient online submission

- thorough peer review by experienced researchers in your field

- rapid publication on acceptance

- support for research data, including large and complex data types

- gold Open Access which fosters wider collaboration and increased citations

- maximum visibility for your research: over $100 \mathrm{M}$ website views per year

At BMC, research is always in progress.

Learn more biomedcentral.com/submissions 